\title{
La FMH à propos du premier paquet visant à freiner les coûts
}

\author{
Nora Wille ${ }^{a}$, Jeanine Glarner ${ }^{b}$, Jürg Schlup ${ }^{c}$ \\ ${ }^{a}$ Dr phil., collaboratrice scientifique du président; ${ }^{b}$ Spécialiste en communication, division Communication de la FMH; ${ }^{c} \mathrm{Dr}$ méd., président de la FMH
}

Suite au rapport du groupe d'experts du Département fédéral de l'intérieur (DFI) présenté en automne 2017, le Conseil fédéral a adopté lors de sa séance du 28 mars 2018 un premier paquet de mesures du programme visant à freiner les coûts de la santé. Les douze mesures prioritaires du Conseil fédéral seront développées au cours des prochains mois et mises en consultation à l'automne 2018. Voici une première analyse.

Le rapport mandaté par le Département fédéral de l'intérieur (DFI) «Mesures visant à freiner la hausse des coûts dans l'assurance obligatoire des soins» [1] avait pour but principal d'analyser les modèles de pilotage et de budgétisation des prestations médicales en Allemagne et aux Pays-Bas [2]. Après six séances et avec l'aide du Secrétariat de l'Office fédéral de la santé publique (OFSP), les experts sélectionnés ont remis leur rapport de 131 pages au chef du DFI, comme convenu à la fin août 2017. Les 38 mesures qui y figurent correspondent en partie aux approches préconisées par la FMH pour freiner les coûts [3]. Par contre, la FMH rejette certaines mesures, et surtout celle proposant d'«instaurer un plafond contraignant pour l'augmentation des coûts de l'AOS» [1]. Cette introduction indirecte d'un budget global obligerait les médecins à prodiguer les soins selon des objectifs politiques (les coûts) plutôt que selon des critères médicaux.

\section{Les priorités du Conseil fédéral dans un premier paquet de mesures}

Le 28 mars 2018, le Conseil fédéral a priorisé neuf mesures sur les 38 du rapport et trois parmi celles proposées par le DFI. Pour la mise en œuvre de ces mesures, il est prévu de les mettre en consultation à l'automne 2018, afin de les examiner dans le cadre d'une révision de la LAMal [4].

\section{Davantage d'innovations et des contrôles plus efficaces}

En tout premier, la mesure MO2 du rapport d'experts est reprise, qui propose l'introduction d'un article ex- périmental pour que «les cantons et les partenaires tarifaires puissent développer des projets novateurs en dehors du cadre de la LAMal pour freiner la hausse des coûts» [4]. La FMH approuve cette démarche visant à encourager des projets pilotes à durée limitée et réversibles, permettant d'évaluer de manière fiable l'impact et les effets secondaires des interventions dans notre

La FMH soutient les mesures visant à freiner la hausse des coûts qui peuvent être mises en place de manière efficace et sans restriction des prises en charge.

système de santé complexe, avant qu'ils ne soient mis en œuvre sur tout le territoire. En plus de l'autorisation du Conseil fédéral ou du département compétent, comme le prévoit le rapport [1] (p. 36), il est indispensable d'associer à ces projets des représentants des fournisseurs de prestations, de chercher leur accord, mais aussi de fixer de manière conjointe et transparente les indicateurs qui permettront de les évaluer. Une autre mesure prioritaire veut renforcer le contrôle des factures (MO9): les assureurs-maladie doivent contrôler plus systématiquement les prestations, vérifier les factures et demander aux fournisseurs de prestations indélicats de rendre des comptes [4]. Si l'«effet souhaité» [4] (qui n'est pas défini plus précisément) n'arrive pas, il est prévu de créer un organe indépendant de contrôle des factures (M35) regroupant les ressources actuelles des assureurs-maladie afin d'offrir des contrôles plus efficaces et plus performants, grâce à des économies d'échelle et des volumes de données plus importants. Sur le fond, la FMH approuve toutes les mesures visant à améliorer l'équité, la comparabi- 
lité et l'efficience des contrôles de factures et permettant, de ce fait, d'éviter les procédures d'économicité injustifiées. Le contrôle par un organisme de droit public, indépendant des caisses-maladie, pourrait être judicieux. Mais les objectifs précis d'un tel organisme et le rapport coût-utilité de son travail (y c. la charge administrative induite pour les fournisseurs de prestations) devraient être fixés à l'avance et réexaminés à intervalles réguliers. Comme le patient joue le rôle le plus important dans ce contrôle, la proposition du DFI d'établir une copie compréhensible de la facture pour le patient doit être soutenue, surtout que l'art. 42 al. 3 LAMal impose déjà depuis 2009 une "facture détaillée et compréhensible».

\section{Conflits de gouvernance des cantons et système de prix de référence}

La mesure M36 «Réduire les conflits de gouvernance des cantons" correspond à la demande de la FMH de réduire les rôles multiples des cantons et d'éviter les conflits d'intérêts et le manque de transparence des subventions [3]. En revanche, il serait insuffisant de ne créer qu'une «instance indépendante d'approbation des tarifs compétente pour les tarifs cantonaux ou au moins pour les tarifs hospitaliers" [4]. La FMH demande aussi que les cantons se retirent de la propriété des hôpitaux [3]. Elle ne comprend pas non plus pourquoi la mesure M03 «Exclure des listes cantonales les hôpitaux proposant des bonus liés à des objectifs quantitatifs et des kick-back», que le groupe d'experts a qualifiée de

Le corps médical ne se tient pas à disposition pour réaliser des souhaits politiques comme le plafonnement des coûts et un rationnement déguisé.

mesure pouvant être immédiatement mise en place, ne fait pas partie de ce premier paquet de mesures.

La FMH se montre par contre critique face au système de prix de référence (M22) prévu pour les médicaments. Un petit marché comme celui de la Suisse comporte le risque de retards réguliers de livraison et d'une restriction de la sécurité de la prise en charge. De plus, l'obligation de changer souvent de médicaments impacterait directement la sécurité des patients.

\section{L'autonomie tarifaire sera-t-elle une nouvelle fois vidée de son sens?}

Sur les douze mesures du Conseil fédéral retenues dans le premier paquet, cinq concernent les tarifs. Pour la mesure M34, qui prévoit une organisation tarifaire nationale pour le secteur ambulatoire, la FMH a déjà manifesté son soutien pour autant que l'autonomie de la structure et de l'organisation reste garantie pour les partenaires tarifaires et que le Conseil fédéral se limite comme aujourd'hui à approuver le tarif proposé [3].

\section{Les compétences subsidiaires rendent l'échec} des négociations trop attractif pour certains des protagonistes et préparent le terrain pour un tarif d'Etat.

Par voie de conséquence, la mesure M25 «Maintenir la structure tarifaire à jour» ne peut être soutenue que si les données exigées sont transmises à l'organisation tarifaire nationale et non au Conseil fédéral.

La FMH rejette catégoriquement que "la structure tarifaire pour les prestations médicales ambulatoires TARMED soit adaptée chaque année par le Conseil fédéral» [1] (p. 79). Pour elle, l'autonomie tarifaire et organisationnelle n'est pas négociable, que ce soit avec le système actuel ou avec une organisation tarifaire nationale paritaire.

De plus, les partenaires tarifaires seront tenus de convenir des forfaits avec les assureurs (M15) dans le domaine ambulatoire. Les assureurs et les fournisseurs de prestations sont favorables à des forfaits ambulatoires et s'attellent déjà à leur développement. Si les forfaits devaient malgré tout être définis dans la loi et associés à une compétence subsidiaire supplémentaire du Conseil fédéral, ils équivaudraient à une nouvelle intervention dans l'autonomie tarifaire [1]. L'exigence de tenir compte des effets d'économie d'échelle dans la structure tarifaire (M16) fait aujourd'hui déjà partie intégrante d'un tarif approprié et conforme aux principes de l'économie d'entreprise selon la LAMal. Ici aussi, vouloir "confier à la Confédération la tâche de gérer le système de façon plus ferme, au moyen de mesures d'accompagnement et de mesures subsidiaires» [1] (p. 63) constituerait une intervention inutile dans l'autonomie tarifaire.

\section{Entrée du budget global par la petite porte}

Dans ce contexte, la mesure «Pilotage des coûts par les partenaires tarifaires» doit être considérée de manière particulièrement critique. En proposant cette mesure, le DFI entend obliger les fournisseurs de prestations et les assureurs à "prévoir des mesures de pilotage des coûts et des prestations dans leurs conventions tarifaires» [4], avec un rôle subsidiaire des cantons et de la Confédération. Ce n'est rien d'autre qu'une manière détournée de mettre en place un budget global, et de déléguer aux partenaires tarifaires la question politi- 
Mesures priorisées par le Conseil fédéral et position de la FMH

\begin{tabular}{|c|c|}
\hline Premières mesures examinées & Position de la FMH \\
\hline Article expérimental (M02) & $\begin{array}{l}\text { OUI, dans la mesure où les fournisseurs de prestations } \\
\text { sont consultés et donnent leur accord }\end{array}$ \\
\hline $\begin{array}{l}\text { Copie de la facture au patient (Proposition } \\
\text { DFI) }\end{array}$ & OUI \\
\hline Renforcer le contrôle des factures (M09) & OUI \\
\hline $\begin{array}{l}\text { Organe indépendant de contrôle des } \\
\text { factures (M35) }\end{array}$ & $\begin{array}{l}\text { OUI, dans la mesure où les objectifs et le rapport coût- } \\
\text { utilité de son travail sont fixés et évalués }\end{array}$ \\
\hline $\begin{array}{l}\text { Réduire les conflits de gouvernance des } \\
\text { cantons (M36) }\end{array}$ & OUI, mais de manière encore plus claire et approfondie \\
\hline Système de prix de référence ( $M 22)$ & $\begin{array}{l}\text { NON à la mise en danger de la sécurité des patients et } \\
\text { de la prise en charge }\end{array}$ \\
\hline Organisation tarifaire nationale (M34) & $\begin{array}{l}\text { OUI, pour autant que l'autonomie de la structure et de } \\
\text { l'organisation reste garantie pour les partenaires } \\
\text { tarifaires }\end{array}$ \\
\hline $\begin{array}{l}\text { Maintenir la structure tarifaire à jour } \\
\text { (M25) }\end{array}$ & $\begin{array}{l}\text { OUI, mais dans le cadre d'une organisation tarifaire } \\
\text { nationale paritaire }\end{array}$ \\
\hline $\begin{array}{l}\text { Forfaits dans le domaine ambulatoire } \\
\text { (M15) }\end{array}$ & OUI aux forfaits - NON à la compétence subsidiaire \\
\hline $\begin{array}{l}\text { Effets d'économie d'échelle dans la } \\
\text { structure tarifaire (M16) }\end{array}$ & $\begin{array}{l}\text { OUI à la prise en compte des effets d'économie } \\
\text { d'échelle - NON à une compétence subsidiaire } \\
\text { supplémentaire }\end{array}$ \\
\hline $\begin{array}{l}\text { Pilotage des coûts par les partenaires } \\
\text { tarifaires (Proposition DFI) }\end{array}$ & NON à l'entrée du budget global par la petite porte \\
\hline
\end{tabular}

quement sensible de baisser les coûts de la santé par un plafonnement budgétaire et un rationnement déguisé des soins. Il est hors de question que le corps médical se mette à disposition pour cela.

\section{Les compétences subsidiaires de la Confé- dération vident le partenariat tarifaire de sa substance - l'organisation tarifaire est-elle la solution?}

L'ensemble du Conseil fédéral a toujours souligné l'importance du maintien du partenariat tarifaire, tout en prévoyant davantage de compétences subsidiaires pour la Confédération si les résultats se faisaient attendre. L'hypothèse selon laquelle la pression exercée ainsi sur les partenaires tarifaires favoriserait le succès des négociations est cependant fausse. Car, dans les faits, la compétence subsidiaire impacte aujourd'hui déjà tellement le partenariat tarifaire qu'elle vide l'autonomie tarifaire de sa substance. Depuis 2014, les échecs des négociations tarifaires se sont traduits par des interventions subsidiaires. Ce mécanisme rend l'échec trop attractif pour certains partenaires tarifaires. Dans ce contexte, ajouter des compétences subsidiaires ne devrait pas encourager le succès de la révision tarifaire, mais, au contraire, la compliquer un peu plus et préparer le terrain pour un tarif d'Etat. Une révision tarifaire réussie et un suivi du tarif durable et approprié exigent donc la création d'une organisation tarifaire nationale paritaire qui se chargerait uniquement de la structure tarifaire, avec donc une séparation stricte avec le prix fixé.

\section{Le corps médical exige une maîtrise des coûts efficace - sans nuire à la prise en charge}

La FMH soutient les mesures visant à freiner la hausse des coûts qui peuvent être mises en place de manière efficace et sans restriction des prises en charge. Elle a communiqué souvent sur ce qui est possible [3,5]. Si les prestations financées de manière solidaire doivent être efficaces, appropriées et conformes au principe de l'économicité [5], les réformes de notre système de santé devraient l'être aussi. Par conséquent, l'utilité et l'impact des mesures proposées par le Conseil fédéral devraient être contrôlés de manière transparente. Pour continuer de garantir l'accès aux soins pour tous et la qualité élevée de notre prise en charge médicale, il est recommandé que la mise en œuvre des mesures visant à freiner la hausse des coûts s'appuient sur les principes éprouvés de l'éthique médicale: «primum non nocere, secundum cavere, tertium sanare».

\section{Références}

1 Rapport du groupe d'experts. Mesures visant à freiner la hausse des coûts dans l'assurance obligatoire des soins; 24 août 2017.

2 DFI/OFSP. Des mesures sont nécessaires pour freiner la hausse des coûts de la santé. Communiqué de presse du 2 septembre 2016

3 Wille N, Glarner J, Schlup J. La FMH à propos de la maîtrise des coûts de santé. Bull Méd Suisses. 2018;99(8):224-6.

4 Département fédéral de l'intérieur (DFI), Office fédéral de la santé publique (OFSP), division Communication et Campagnes (29 mars 2018). Mesures visant à freiner la hausse des coûts dans l'assurance obligatoire des soins (AOS): les premières mesures à examiner.

5 Position de la FMH sur la santé publique en Suisse. Berne, octobre 2016 